\title{
Understanding the dust properties in nearby galaxies
}

\author{
Marjorie Decleir ${ }^{1 *}$, Maarten Baes ${ }^{1}$, Ilse De Looze ${ }^{1,2}$, Peter Camps $^{1}$ \\ ${ }^{1}$ Sterrenkundig Observatorium, Universiteit Gent, Belgium \\ ${ }^{2}$ University College of London, United Kingdom
}

\begin{abstract}
Dust is a crucial component in the interstellar medium of galaxies. It regulates several physical and chemical processes. Dust grains are also efficient at absorbing and scattering ultraviolet/optical photons and then re-radiating the absorbed energy in the infrared/submm wavelength range. The spatial distribution and properties of dust in galaxies can hence be investigated in two complementary ways: by its attenuation effects at short wavelengths, and by its thermal emission at long wavelengths. Both approaches have their advantages and challenges. In this contribution, we discuss a number of recent interesting results on interstellar dust in nearby galaxies, obtained by our research group at Ghent University.
\end{abstract}

\section{Introduction}

Interstellar dust only makes up a very small fraction (typically 1\%) of the mass of the interstellar medium in galaxies. However, its impact on the other baryonic components in galaxies should not be underestimated, since dust regulates several physical and chemical processes. For example, dust particles act as a catalyst for the formation of molecular hydrogen, regulate the heating of the neutral gas component through photoelectric heating and inelastic interactions, and provide shielding for molecules from the ultraviolet (UV) radiation of young stars. Furthermore, dust is a reservoir of metals, which are the main building blocks for planet formation, and in fact for all organic material that we know today, including ourselves.

On galaxy-wide scales, interstellar dust also significantly affects our view on galaxies: on average, about 30 to $50 \%$ of the starlight (UV and optical radiation) in the Universe is absorbed by interstellar dust grains, and converted to far-infrared (FIR) and submillimeter (submm) emission (Xu \& Buat 1995; Popescu \& Tuffs 2002; Davies et al. 2012). It is hence important to carefully trace and map the dust in galaxies of different types and in different environments.

In this contribution we discuss some of the progress that our research group has recently made in understanding the distribution and characteristics of interstellar dust in nearby galaxies. We particularly focus on the results of dust radiative transfer modelling of galaxies.

*e-mail: Marjorie.Decleir@UGent.be 


\section{Dust radiative transfer}

One of the ways to investigate the amount and spatial distribution of interstellar dust in galaxies is by analysing its effect on the starlight at UV and optical wavelengths. The proper way to do this is by means of radiative transfer (RT) calculations that take into account the relevant effects (absorption and multiple anisotropic scattering) in a realistic geometrical setting.

The full dust RT equation can be written as (see Steinacker et al. 2013, Eq. 10):

$$
\begin{aligned}
\frac{\mathrm{d} I}{\mathrm{~d} s}(\mathbf{x}, \mathbf{n}, \lambda)=-\kappa_{\mathrm{ext}}(\mathbf{x}, \lambda) \rho(\mathbf{x}) I(\mathbf{x}, \mathbf{n}, \lambda)+ & j_{*}(\mathbf{x}, \mathbf{n}, \lambda)+j_{\mathrm{d}}(\mathbf{x}, \lambda) \\
& +\kappa_{\mathrm{sca}}(\mathbf{x}, \lambda) \rho(\mathbf{x}) \int_{4 \pi} \phi\left(\mathbf{n}, \mathbf{n}^{\prime}, \mathbf{x}, \lambda\right) I\left(\mathbf{x}, \mathbf{n}^{\prime}, \lambda\right) \mathrm{d} \Omega^{\prime}
\end{aligned}
$$

The left-hand side of this equation represents the change in intensity of radiation $I$, at a certain wavelength $\lambda$, over an infinitesimal distance $\mathrm{d} s$ along the path determined by the position $\mathbf{x}$ and the propagation direction $\mathbf{n}$. The different terms in the right-hand side of the equation represent the sinks and sources, i.e., the different physical processes that are responsible for extinction and emission of radiation. From left to right the different terms are: the extinction (including both absorption and scattering) with $\kappa_{\text {ext }}(\mathbf{x}, \lambda)$ the mass extinction coefficient and $\rho(\mathbf{x})$ the mass density; the primary stellar emission $j_{*}(\mathbf{x}, \mathbf{n}, \lambda)$; the thermal dust emission $j_{\mathrm{d}}(\mathbf{x}, \lambda)$; and finally, the scattering source term with the scattering coefficient $\kappa_{\text {sca }}(\mathbf{x}, \lambda)$ and the scattering phase function $\phi\left(\mathbf{n}, \mathbf{n}^{\prime}, \mathbf{x}, \lambda\right)$, describing the probability that a photon originally propagating in direction $\mathbf{n}^{\prime}$ and scattered at position $\mathbf{x}$, will have $\mathbf{n}$ as its new propagation direction after the scattering event.

3D RT is generally considered to be one of the grand challenges in computational astrophysics (Steinacker et al. 2013). The main reason is that the underlying physical processes combine, in the stationary case, to a non-local and non-linear 6D problem. The RT equation (Eq. 1) is an integrodifferential equation that is usually non-linearly coupled to other equations. Solving such a highdimensional non-local, non-linear problem requires substantial computational resources, affecting the solution algorithms and potentially limiting the model complexity.

In spite of the difficulties described above, significant progress has been made in the past decade, with an increasing number of codes capable of dealing with the complete 3D dust RT problem. Furthermore, there has been a huge increase in computing power and a continuous refinement/improvement of the existing codes during the last years. A prominent state-of-the-art code is the Monte Carlo based RT code SKIRT ${ }^{1}$ (Baes et al. 2003, 2011; Camps \& Baes 2015), developed by our research group at Ghent University.

\subsection{Inverse radiative transfer}

RT codes are powerful tools to systematically investigate the effects of attenuation on galaxy models. Often, however, we are interested in the dust content of a specific galaxy based on a set of UV/optical/near-infrared (NIR) images, and an RT code by itself, no matter how advanced, is not sufficient to achieve this. This so-called inverse radiative transfer modelling of galaxies requires a combination of an RT code and an optimisation procedure.

\footnotetext{
${ }^{1}$ www.skirt.ugent.be
} 
Several fitting codes have been set up to combine an RT code with an optimisation algorithm (Xilouris et al. 1997; Bianchi 2007; Schechtman-Rook et al. 2012). This technique has proven to be rather challenging. The star-dust geometry can be quite complex and causes a large number of parameters that need to be fitted: the intrinsic geometry of the star and dust distribution, but also the orientation of both components, and the intrinsic properties of the dust itself. In addition, the run-time and memory requirements for a single simulation are already quite considerable. Running thousands of simulations to obtain the best fit requires a lot of computing resources.

Our research group successfully combined SKIRT with an optimisation library based on genetic algorithms into a code (FitSKIRT) that can simultaneously fit arbitrary 3D models to a set of UV, optical and NIR images (De Geyter et al. 2013, 2014). This "oligochromatic" fitting has clear advantages over standard "monochromatic" fitting, as it can minimise the degeneracies that are hard to overcome when RT models are fitted solely to a single band. De Geyter et al. 2014 describes a full analysis of the unicity of the obtained models, and the technique that is used to explore the large parameter space, avoiding that the code ends up in local minima. To the best of our knowledge, FitSKIRT always finds the best solution in the multi-dimensional parameter space. This technique has been successfully applied to several galaxies (Viaene et al. 2015; De Geyter et al. 2014; De Looze et al. 2012).

Fig. 1 shows the results that were obtained by Decleir et al. (in prep.) for the edge-on spiral galaxy NGC 5866. Edge-on spiral galaxies have been a preferred class for RT modelling, as the dust shows up nicely as a thin dust lane. It can be seen that for the different bands the images on the left are reproduced very well by the models in the middle. On the right, the residuals give an indication about the quality of the fit. The main conclusion derived from modelling this and other edge-on galaxies is that the dust is generally distributed in an extended and thin disk with modest face-on optical depths, as also shown in the pioneering work in this field by Kylafis \& Bahcall (1987) and Xilouris et al. (1997, 1998, 1999).

On the other hand, modelling face-on galaxies needs a somewhat different approach. In Fig. 2, images and models are compared for the face-on galaxy M51. In De Looze et al. (2014) a new technique has been developed to model the RT effects in nearby face-on galaxies. A high-resolution 3D RT model has been constructed with SKIRT to account for the absorption, scattering, and nonlocal thermal equilibrium emission of dust in M51. The 3D distribution of stars has been derived from the 2D morphology observed in the IRAC $3.6 \mu \mathrm{m}$, GALEX far-UV (FUV), H $\alpha$, and MIPS 24 $\mu \mathrm{m}$ wavebands, assuming an exponential vertical distribution with an appropriate scale height. The dust geometry has been constrained through the FUV attenuation, which has been derived from the observed total-IR-to-FUV luminosity ratio. The stellar luminosity, star formation rate (SFR), and dust mass have been scaled to reproduce the observed stellar spectral energy distribution (SED), FUV attenuation, and IR SED.

\subsection{DustPedia}

It would be useful to have a more consistent way of investigating a large sample of galaxies in the same way as was done for some individual galaxies. To this aim, the DustPedia ${ }^{2}$ project was launched. DustPedia is an EU-FP7 funded project and its primary goal is to make the most extensive study of dust in local galaxies. More specifically, multi-wavelength images from the UV to the mm, including Herschel and Planck data, are combined for a sample of over 800 galaxies in the nearby Universe.

\footnotetext{
${ }^{2}$ www.dustpedia.com
} 


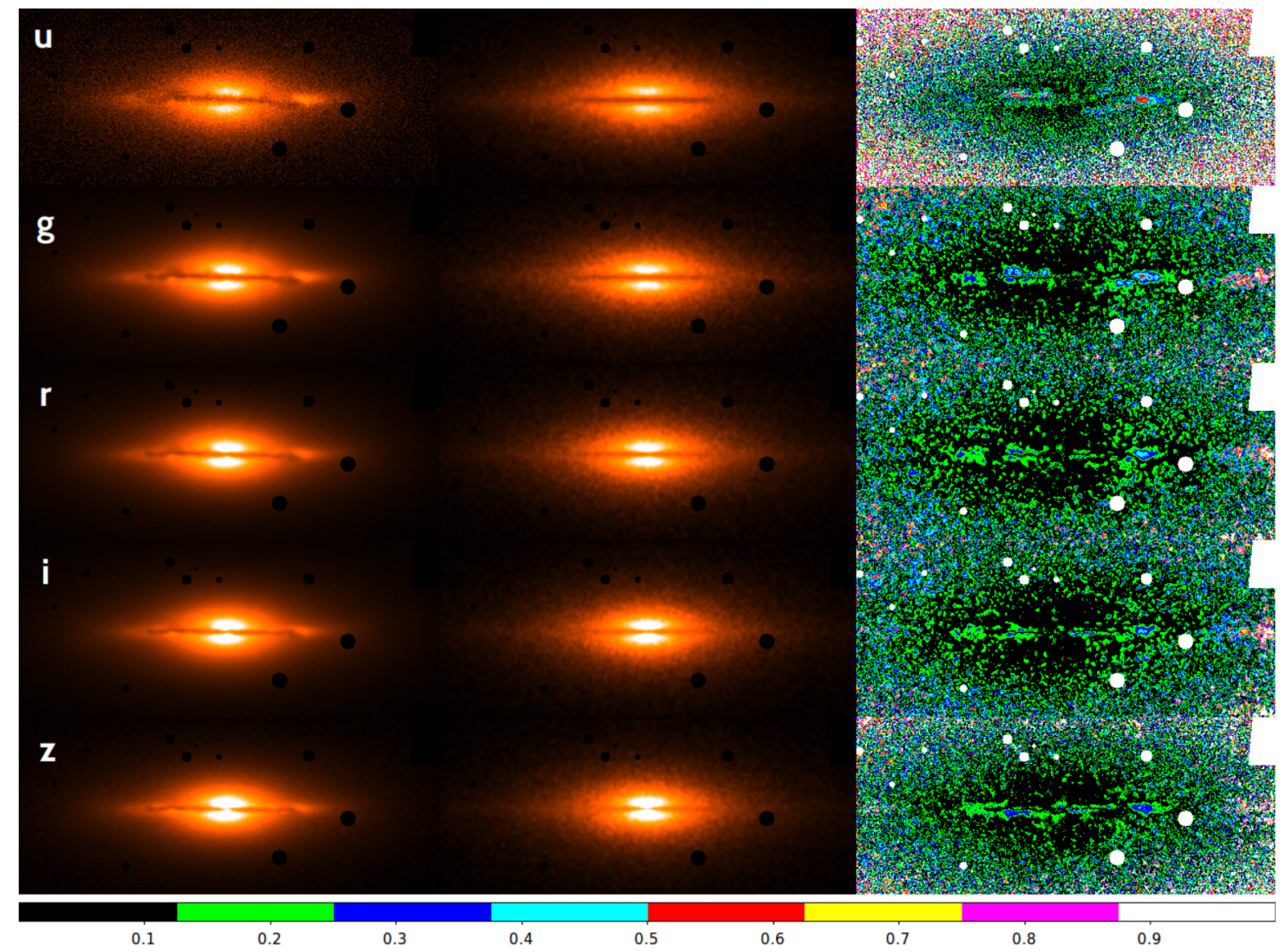

Figure 1: Results of the oligochromatic FitSKIRT RT fits to the edge-on spiral galaxy NGC 5866. The left-hand column represents the observed images in the $\mathrm{u}, \mathrm{g}, \mathrm{r}, \mathrm{i}$ and $\mathrm{z}$ bands (from top to bottom), and the middle column contains the corresponding fits in the same bands. The right-hand column contains the residual images, which indicate the relative deviation between the fit and the image. A colour bar with the scaling of the latter is indicated at the bottom. Including calibration, background and Poisson noise, we derive total uncertainties that range between 5 to $10 \%$ across the image in all SDSS bands (Decleir et al. in prep.)

These galaxies are being modelled and analysed with state-of-the art software codes and tools.

Their archive, which will be made public at the end of the project, contains the full set of images and derived model parameters of all galaxies. However, some edge-on galaxies of the sample lack good optical SDSS observations. In this prospect, it could be useful to observe these galaxies with the optical imager at the $3.6 \mathrm{~m}$ Devasthal Optical Telescope (DOT). The images could be used to model a large sample of edge-on galaxies with FitSKIRT.

\section{DustKING}

One of the most important properties of the dust is the way it attenuates the starlight, because the dust attenuation is a crucial parameter in the determination of several intrinsic galaxy properties, such as the masses of the stellar populations, the SFRs and the star formation histories (SFH). Unfortunately, the dust attenuation is still a very uncertain parameter and our knowledge about the dust properties is limited to our own Galaxy and the Magellanic Clouds. Therefore, in extragalactic studies, it is usually assumed that the shape of the dust attenuation curve is similar as observed in the Milky Way (MW). 

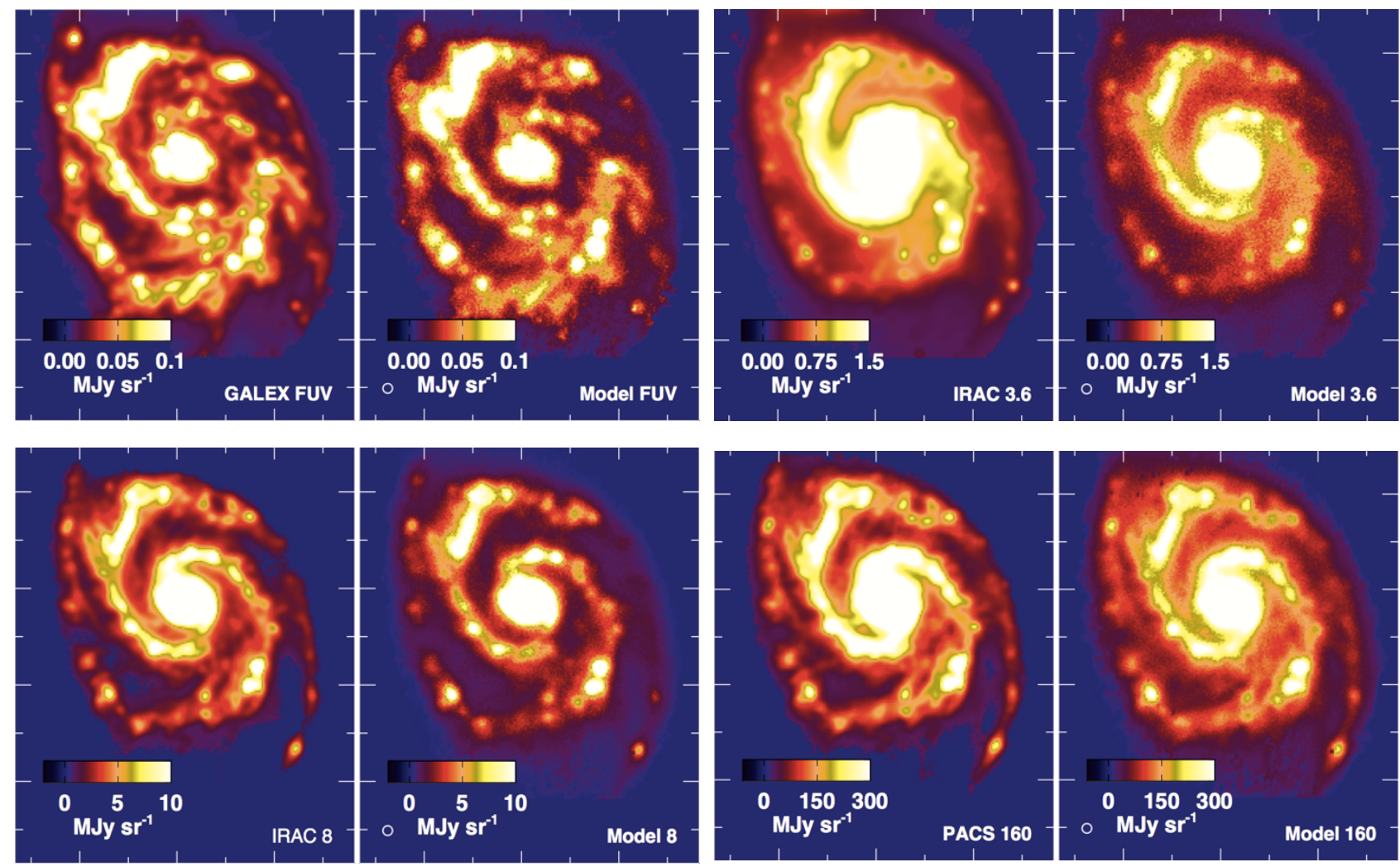

Figure 2: Observed (left) and modelled (right) images of M51 for the GALEX FUV (top left), IRAC $3.6 \mu \mathrm{m}$ (top right), IRAC $8 \mu \mathrm{m}$ (bottom left) and PACS $160 \mu \mathrm{m}$ (bottom right) wavebands. The different wavebands give us information about the contribution to the radiation of different components of the galaxy. For example, the FUV image indicates where the young stars are situated and from the model we can derive how much radiation they emit. The NIR IRAC $3.6 \mu \mathrm{m}$ image gives information about the more evolved stars. The MIR IRAC $8 \mu$ m image shows the radiation by the hot dust, while the FIR PACS $160 \mu \mathrm{m}$ image reveals the location of the cool dust (De Looze et al. 2014)

However, Fig. 3 indicates that there are clear deviations from the "universal" dust attenuation law of the MW.

To overcome this problem, we initiated an ambitious program, DustKING, that aims to investigate the variations in the shape of dust attenuation curves and dust properties in the nearby Universe. To this aim, we use the combined SINGS and KINGFISH samples, containing 79 nearby galaxies with a wide variety of morphological galaxy types, metal abundances and star formation activities.

We use multi-wavelength data from the FUV to the submm. We are focusing on UV data from both the GALEX and the SWIFT missions. The UV filters on the SWIFT space telescope uniquely cover the $2175 \AA$ bump feature, which will allow us to constrain the strength of this bump feature. Since the standard data reduction pipeline was not optimised for extended sources, we created a new pipeline to reduce the SWIFT data (Decleir et al. in prep.). This pipeline has been used to reduce the data of the spiral galaxy NGC 628.

Fig. 4 shows a first result obtained from the UV data. In this figure, we show six color plots where one UV color is plotted against another UV color. These color-color plots are a first step to compare models with observations and give an idea about the stellar population and the level of attenuation in the galaxy. The black dots are the data obtained from the images, whereas the lines are theoretical 


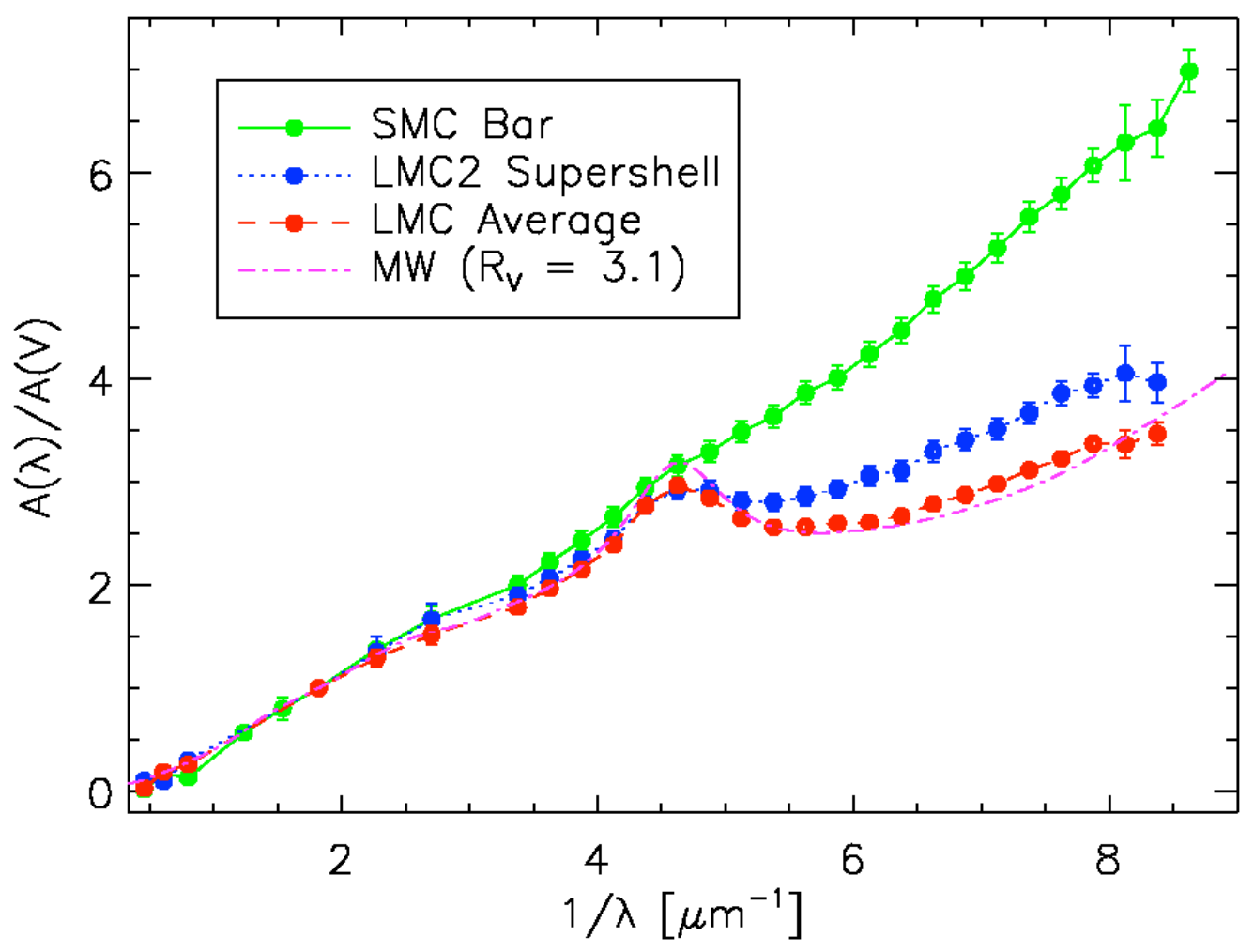

Figure 3: The variety of dust attenuation curves observed in the Milky Way (MW) and the Magellanic Clouds $($ Small/Large $=$ SMC/LMC) $($ Gordon et al. 2003)

curves obtained by attenuating the spectra of single stellar populations assuming different dust extinction laws and levels of dust attenuation. The reddish lines correspond to the Calzetti attenuation law, the blue lines are for the extinction in the MW with $\mathrm{R}_{V}=3.1$ and the greenish lines correspond to the extinction curve in the Small Magellanic Cloud (SMC). These models occupy different regions of the color diagrams, depending on the assumed attenuation/extinction curve and thus give us an idea about the most applicable extinction curve and the properties of the dust in the galaxy.

If we have a more careful look at these plots, we find that the observed UV colors appear most consistent with a MW extinction curve, or maybe a Calzetti attenuation curve as the bottom left plot would suggest. However, they do not at all agree with the SMC curve. From this we could cautiously derive that the UV slope of the attenuation curve that best fits for NGC 628 is rather shallow and not as steep as for the SMC. This would in turn mean that the dust properties in NGC 628 seem to be consistent with the dust properties in the MW. Furthermore, we indicated stellar populations with ages of 1,10 and $100 \mathrm{Myr}$. As we are looking at UV colors, we expect the light to be dominated by young stars of a few $100 \mathrm{Myr}$ or younger, which is indeed confirmed by the data points in the plots.

The assumption of a specific single stellar population is not very realistic. Therefore, we are now using a more advanced approach by fitting analytic functions to the observed SEDs, taking into account the SFH and combining observations at different wavelengths in order to characterize the complete stellar population in NGC 628. Doing this, we are able to quantify the slope of the attenuation curve in the UV wavelength regime and the width and amplitude of the $2175 \AA$ absorption feature. To this aim, we construct SED models including stellar emission and dust obscuration, which requires optical data. About 20 galaxies of the SINGS/KINGFISH sample lack good optical data. It 


\section{SWIFT/GALEX color-color plots}

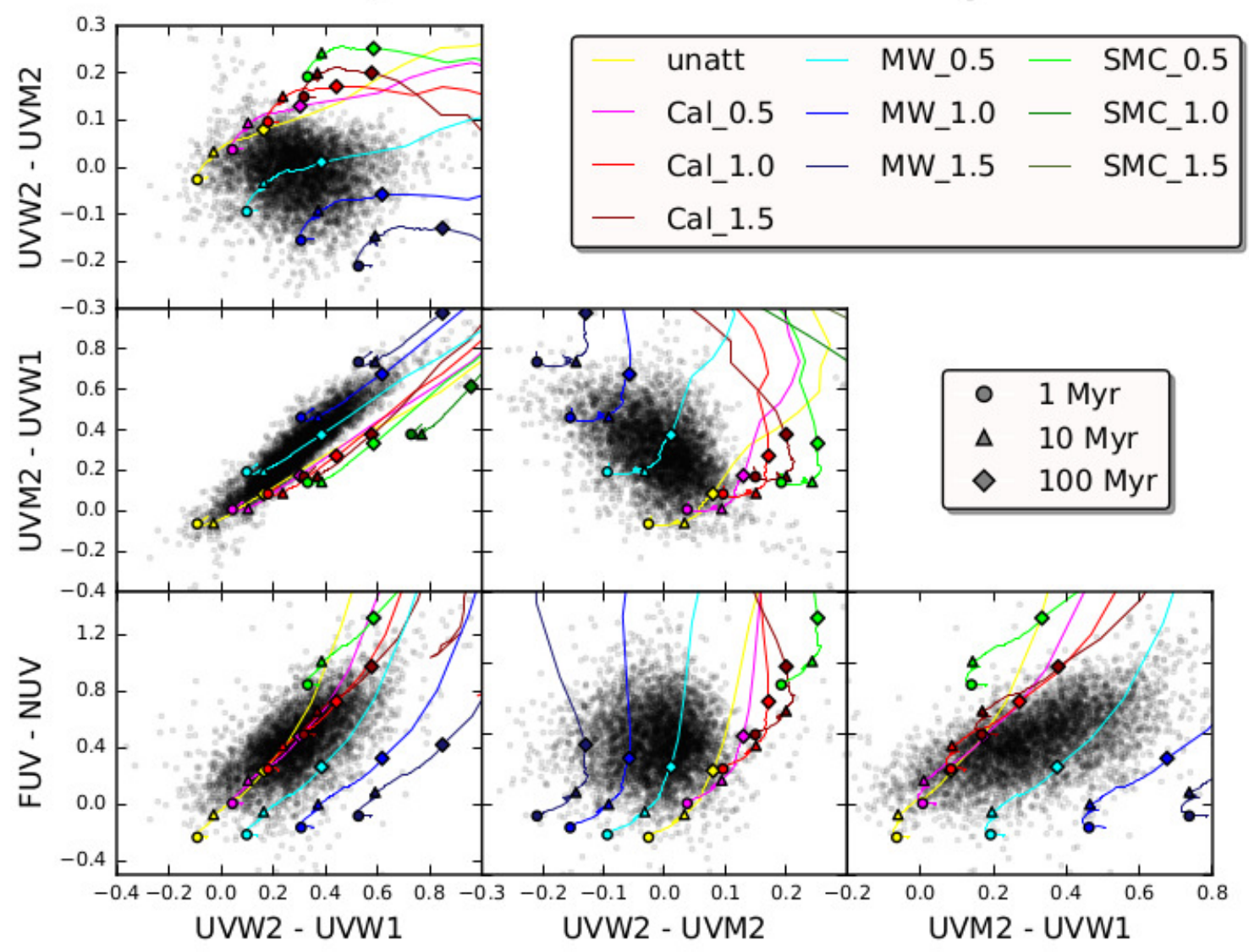

Figure 4: SWIFT/GALEX color-color plots for NGC 628. The black dots are the data obtained from the images, whereas the lines are theoretical curves obtained by attenuating the spectra of single stellar populations assuming different dust extinction laws and levels of dust attenuation. Furthermore, we indicated stellar populations with ages of $1 \mathrm{Myr}$ (circles), $10 \mathrm{Myr}$ (triangles) and $100 \mathrm{Myr}$ (diamonds). For more details: see section 3 (Decleir et al. in prep.)

would thus be useful to obtain the missing data with the CCD imager or with the FOSC instrument on the $3.6 \mathrm{~m}$ DOT which has ugriz filters. Since the u-filter on this imager is particularly sensitive, it would be useful to have good u-band data to constrain the attenuation curve and SED in the gap between the UV and optical data.

\section{Conclusions}

This contribution can be summarised as follows:

- Dust is an important ingredient of the interstellar medium. It can be traced by its attenuation effects on the starlight, or by its direct thermal emission at infrared and submm wavelengths. $3 \mathrm{D}$ dust radiative transfer modelling allows a self-consistent analysis of dust extinction and emission.

- Recent years have seen an enormous progress in the field of 3D dust radiative transfer, thanks to an increase in computing power, the development and maturing of different algorithms, and 
a wealth of multi-wavelength data.

- Thanks to the coupling of radiative transfer codes to optimisation routines, detailed 3D radiative transfer modelling of individual galaxies has become possible.

- In the DustKING project we aim to investigate the different shapes of the attenuation curves of nearby galaxies. SWIFT UV data will be used to characterize the $2175 \AA$ absorption bump. The first results for NGC 628 suggest that the dust properties in this galaxy are similar to those in the Milky Way.

\section{Acknowledgements}

This research is supported financially in part by the Flemish Fund for Scientific Research (FWOVlaanderen). We like to thank the organisers of the first BINA workshop for their hospitality.

\section{References}

Baes M., Davies J. I., Dejonghe H. et al. 2003, MNRAS, 343, 1081

Baes M., Verstappen J., De Looze I. et al. 2011, ApJS, 196, 22

Bianchi S. 2007, A\&A, 471, 765

Camps P., Baes M. 2015, A\&C, 9, 20

Davies J. I., Bianchi S., Cortese L. et al. 2012, MNRAS, 419, 3505

De Geyter G., Baes M., Fritz J., Camps P. 2013, A\&A, 550A, 74

De Geyter G., Baes M., Camps P. et al. 2014, MNRAS, 441, 869

De Looze I., Baes M., Bendo G. J. et al. 2012, MNRAS, 427, 2797

De Looze I., Fritz J., Baes M. et al. 2014, A\&A, 571A, 69

Gordon K. D., Clayton G. C., Misselt K. A., Landolt A. U., Wolff M. J. 2003, ApJ, 594, 279

Kylafis N. D., Bahcall J. N. 1987, ApJ, 317, 637

Popescu C. C., Tuffs R. J. 2002, MNRAS, 335L, 41

Schechtman-Rook A., Bershady M. A., Wood K. 2012, ApJ, 746, 70

Steinacker J., Baes M., Gordon K. D. 2013, ARA\&A, 51, 63

Viaene S., De Geyter G., Baes M. et al. 2015, A\&A, 579A, 103

Xilouris E. M., Kylafis N. D., Papamastorakis J., Paleologou E. V., Haerendel G. 1997, A\&A, 325, 135

Xilouris E. M., Alton P. B., Davies J. I. et al. 1998, A\&A, 331, 894

Xilouris E. M., Byun Y. I., Kylafis N. D., Paleologou E. V., Papamastorakis J. 1999, A\&A, 344, 868

Xu C., Buat V. 1995, A\&A, 293L, 65 